\title{
Comparison of Harmonic scalpel and Ligasure devices in laparoscopic Roux-en-Y gastric bypass
}

\author{
Serdar Kirmizi, Cuneyt Kayaalp, Servet Karagul, Ali Tardu, Ismail Ertugrul, Fatih Sumer, Mehmet Ali Yagci \\ Department of Gastrointestinal Surgery, Faculty of Medicine, Inonu University, Malatya, Turkey \\ Videosurgery Miniinv 2017; 12 (1): 28-31 \\ DOI: https://doi.org/10.5114/wiitm.2017.66641
}

\begin{abstract}
Introduction: Laparoscopic Roux-en-Y gastric bypass (LRYGB) is one of the most preferred bariatric procedures in the world for surgical treatment of morbid obesity. The Harmonic scalpel (HS) and LigaSure (LS) are the most commonly used devices in laparoscopic surgery. As far as we know, there is no comparative study of the two energy devices in LRYGB for morbid obesity.

Aim: To compare the intraoperative performances of the two energy devices in LRYGB for morbid obesity.

Material and methods: The HS and LS were used in 43 and 42 cases, respectively. The patient demographics of both groups were comparable. The duration of the procedures (gastric pouch creation time and total operation time), quantity of bleeding (during gastric pouch creation and total quantities of bleeding) and the number of pneumoperitoneum desufflations due to smoking that impaired sight fields were recorded prospectively.

Results: Gastric pouch creation time (HS: $22.5 \pm 9.5$ vs. LS: $19.5 \pm 9.7 \mathrm{~min}, p=0.15$ ), bleeding during gastric pouch preparation (HS: $15.3 \pm 30.5$ vs. LS: $17.5 \pm 31.3 \mathrm{ml}, p=0.74$ ), total operation time (HS: $183.2 \pm 47 \mathrm{vs}$. LS: $165.3 \pm 37.1 \mathrm{~min}$, $p=0.06$ ) and total bleeding (HS: $110 \pm 195.5$ vs. LS: $102.5 \pm 70 \mathrm{ml}, p=0.81$ ) were similar in the two groups. Only the mean number of pneumoperitoneum desufflations due to smoking was lower in the HS group (HS: $0.28 \pm 0.49$ vs. $L S: 0.57 \pm 0.78, p=0.04$ ).
\end{abstract}

Conclusions: The HS and LS performed similarly in $\angle R Y G B$, with fewer desufflations from smoking in the HS group.

Key words: gastric bypass, laparoscopic, obesity surgery, surgical stapling.

\section{Introduction}

Laparoscopic Roux-en-Y gastric bypass (LRYGB) is one of the most preferred bariatric procedures in the world for surgical treatment of morbid obesity [1]. Developments in bariatric surgery have recently increased in parallel with the developments in the energy devices that are the inevitable tools for laparoscopic surgery. The Harmonic scalpel (HS) and LigaSure (LS) are the most commonly used devices in laparoscopic surgery. They enable vascular closure and tissue dissection by using different types of energy, and each has its advantages and disadvantages.
As far as we know, there is no comparative study of the two energy devices in LRYGB for morbid obesity.

\section{Aim}

The aim of this study was to compare the intraoperative performance of HS and LS (5 $\mathrm{mm})$ in LRYGB.

\section{Material and methods}

We applied to the Inonu University Faculty of Medicine Ethical Board and obtained approval for this study (Approval No. 2014-105). This prospective study was also registered with clinicaltrials.gov 
(NCT02538328). The lack of previous studies on LRYGB made it impossible to find out an "expected frequency" that is necessary to calculate the sample size by power analysis. A statistician suggested a total of 90 patients (45 in each group) and intermittent evaluations of the outputs. Between October 2014 and August 2015, 90 patients in total were included after their informed consent had been obtained. Before the study was launched, the surgeons involved had overall experience with more than 500 cases of gastric bypasses with both devices. In the present study, HS Ace (Ethicon Endo-Surgery, Johnson \& Johnson, Cincinnati, OH, USA) was used in 45 cases of patients who underwent surgery in the first 5-month period, and LS (Valleylab Healthcare Group LP, Boulder, CO, USA) was used in 45 cases in the next 5 -month period. Consecutive grouping was used instead of a randomised study because the energy devices were purchased at different times. A standardised LRYGB technique was applied to all patients [2]. Patients were assessed by collecting their data, including their age, sex, height, weight, body mass index (BMI), American Society of Anesthesiologists (ASA) score and co-morbidities. Gastric pouch preparation time, quantity of bleeding during gastric pouch preparation, number of pneumoperitoneum desufflations due to smoking and the total quantities of bleeding and total operation time were recorded. Data collection was not regularly arranged by a specific person. Measurements were collected sometimes by the anesthesiologists (blood loss in the aspirator container), by the operating room nurse, by a surgeon in the procedure or by a surgeon in the operating room but not in the procedure.

\section{Statistical analysis}

Data were analysed using the Student $t$-test and $\chi^{2}$ test. $P$-value $<0.05$ was considered significant.

\section{Results}

We planned for 90 cases, but data were not complete in 5 cases that were excluded and the remaining 85 patients were analyzed, 43 in the HS and 42 in the LS group. No difference was observed for age, sex, height, weight, BMI, co-morbidities or ASA score between groups (Table I). When the intraoperative values were compared, the mean number of desufflations due to smoking was higher in LS (HS: 0.28 \pm 0.49 vs. LS: $0.57 \pm 0.78, p=0.04)$. Gastric pouch preparation time (HS: $22.5 \pm 9.5$ vs. LS: $19.5 \pm 9.7 \mathrm{~min}$, $p=0.15)$, quantity of bleeding during gastric pouch preparation (HS: $15.3 \pm 30.5$ vs. LS: $17.5 \pm 31.3 \mathrm{ml}$, $p=0.74$ ), and total quantities of bleeding (HS: 110 \pm 195.5 vs. LS: $102.5 \pm 70 \mathrm{ml}, p=0.81$ ) were similar in both groups. Total operation time did not show statistical significance but was slightly shorter in the LS group (HS: $183.2 \pm 47$ vs. LS: $165.3 \pm 37.1 \mathrm{~min}$, $p=0.06)$.

\section{Discussion}

In LRYGB, there are many methods for controlling bleeding and reliable dissection, such as energy devices, electrocautery, clips, vascular staplers and intracorporeal sutures [3]. Electrocautery is cost-effective and easy to access and therefore commonly preferred. However, it provides insufficient haemostasis when compared to bipolar or ultrasonic energy devices and causes more lateral thermal damage in the peripheral tissues [4].

The HS is based on the mechanism of ultrasound, while LS uses electrothermal bipolar technology by closing vessels up to $7 \mathrm{~mm}$ in diameter [5]. The LS applies low voltage by using a high electrical current and denatures the elastin and collagen at the vascular wall, providing tissue coagulation. Pressure applied by hand also contributes to closure of the vessels mechanically. The HS enables protein denaturation within the vessel by means of ultrasonic vibrations and closes and cuts vessels up to $5 \mathrm{~mm}$ in diameter [6]. Although vessel sealing time with HS is dependent on the user, the sealing times of both devices are similar [7]. After activation, tip temperature is higher in HS, but less peripheral tissue damage has been reported for HS [7].

We use both devices in our own clinic when available. Due to our hospital cost policy, usually only one of these energy devices is available in the operating theatre. This is why the present study was planned not as a randomised study but as a consecutive study. Many studies in the literature have compared the efficiency of these two devices in endocrine, colorectal and hepatobiliary surgery, but randomised studies are very limited [8-10]. Campagnacci et al. [9] found no difference in the duration for colorectal surgery between the two devices, but there was less bleeding with LS. Rimonda et al. [10] did not observe any difference between the operation times 
Table I. Demographic characteristics of the patients

\begin{tabular}{|c|c|c|c|}
\hline Parameters & Harmonic scalpel $(n=43)$ & LigaSure $(n=42)$ & $P$-value \\
\hline Age & $39.2 \pm 9.9$ & $36.7 \pm 11.0$ & NS \\
\hline Gender: & & & NS \\
\hline Male & 9 & 14 & \\
\hline Female & 34 & 28 & \\
\hline Height $[\mathrm{cm}]$ & $164.9 \pm 6.8$ & $166.0 \pm 8.3$ & NS \\
\hline Weight [kg] & $125.3 \pm 16.3$ & $129.3 \pm 19.3$ & NS \\
\hline BMI $\left[\mathrm{kg} / \mathrm{m}^{2}\right]$ & $45.8 \pm 5.9$ & $45.2 \pm 6.5$ & NS \\
\hline ASA score: & & & NS \\
\hline 1 & 3 & 4 & \\
\hline$\|$ & 29 & 31 & \\
\hline III & 11 & 7 & \\
\hline Comorbidities (total): & & & NS \\
\hline Yes & 17 & 20 & \\
\hline No & 26 & 22 & \\
\hline Diabetes mellitus: & & & NS \\
\hline Yes & 15 & 11 & \\
\hline No & 28 & 31 & \\
\hline Hypertension: & & & NS \\
\hline Yes & 9 & 10 & \\
\hline No & 34 & 32 & \\
\hline Lung diseases: & & & NS \\
\hline Yes & 1 & 3 & \\
\hline No & 42 & 39 & \\
\hline
\end{tabular}

$B M I$ - body mass index, ASA - American Society of Anaesthesiologists, NS - not significant.

of the two devices in colorectal surgery [10]. In bariatric surgery, Tsamis et al. compared these devices in laparoscopic sleeve gastrectomy [8]. The authors reported that operation durations were similar (HS: $40 \pm 20$ vs. LS $45 \pm 15$ min, $p=0.20$ ), but no data were reported on the technical details or smoking deficits of the devices. In sleeve gastrectomy, energy devices are particularly used only for releasing the greater curvature of the stomach, whereas in LRYGB, the devices are used in many fields such as gastric pouch preparation, dividing the intestinal mesentery, gastrostomy, enterotomy and dividing the greater omentum. The LRYGB can be a good option for comparing energy devices in different areas of dissection and haemostasis. This study marks the first time these two popular energy devices have been compared for LRYGB. We did not find any differences between the two groups with respect to the gastric pouch preparation time, quantity of bleeding during gastric pouch preparation, total quantity of bleeding or total duration of surgery. We only found that there was more smoking with LS than with HS. Some smoking occurs with all sealing and dissection devices. Impairment of the sight field due to this is a serious problem and requires desufflation sometimes. A higher rate of smoking with LS was reported by Vilos and Rajakumar in 2013 [11], and we confirm their findings. 


\section{Conclusions}

The HS and LS yielded similar clinical data in LRYGB. The HS had lower smoking-induced desufflation rates. The selection of the energy devices should be based on the preference of the surgeon and the condition of the hospital.

\section{Conflict of interest}

The authors declare no conflict of interest.

\section{References}

1. Linkov F, Bovbjerg DH, Freese KE, et al. Bariatric surgery interest around the world: what Google Trends can teach us. Surg Obes Relat Dis 2014; 10: 533-8.

2. Kayaalp C, Sumer F, Abdullayev A. Laparoscopic Roux-en-Y gas tric bypass. Eur J Endosc Laparosc Surg 2014; 1: 196-204.

3. Seehofer D, Mogl M, Boas-Knoop S, et al. Safety and efficacy of new integrated bipolar and ultrasonic scissors compared to conventional laparoscopic 5-mm sealing and cutting instruments. Surg Endosc 2012; 26: 2541-9.

4. Diamantis T, Kontos M, Arvelakis A, et al. Comparison of mo nopolar electrocoagulation, bipolar electrocoagulation, ultracision, and ligasure. Surg Today 2006; 36: 908-13.

5. Sankaranarayanan G, Resapu RR, Jones DB, et al. Common uses and cited complications of energy in surgery. Surg Endosc 2013; 27: 3056-72.

6. Lyons SD, Law KS. Laparoscopic vessel sealing technologies. J Minimally Invasive Gynecol 2013; 20: 301-7.

7. Person B, Vivas DA, Ruiz D, et al. Comparison of four energy-based vascular sealing and cutting instruments: a porcine model. Surg Endosc 2008; 22: 534-8.

8. Tsamis D, Natoudi M, Arapaki A, et al. Using Ligasure ${ }^{\mathrm{TM}}$ or Harmonic Ace in laparoscopic sleeve gastrectomies? A prospective randomized study. Obes Surg 2015; 25: 1454-7.

9. Campagnacci R, De Sanctis A, Baldarelli M, et al. Electrothermal bipolar vessel sealing device vs. ultrasonic coagulating shears in laparoscopic colectomies: a comparative study. Surg Endosc 2007; 21: 1526-31.

10. Rimonda R, Arezzo A, Garrone C, et al. Electrothermal bipolar vessel sealing system vs. harmonic scalpel in colorectal laparoscopic surgery: a prospective, randomized study. Dis Colon Rectum 2009; 52: 657-61.

11. Vilos GA, Rajakumar C. Electrosurgical generators and monopolar and bipolar electrosurgery. J Minim Invasive Gynecol 2013; 20: 279-87.

Received: 16.10.2016, accepted: 25.02.2017. 